\title{
Article
}

\section{Old and new major mergers in the SOSIMPLE galaxy, NGC 7135}

Davison, Thomas, Kuntschner, Harald, Husemann, Bernd, Norris, Mark, Dalcanton, Julianne J, De Rosa, Alessandra, Duc, Pierre-Alain, Bianchi, Stefano, Capelo, Pedro R and Vignali, Cristian

Available at http://clok.uclan.ac.uk/36582/

Davison, Thomas, Kuntschner, Harald, Husemann, Bernd, Norris, Mark ORCID: 0000-0002-7001-805X, Dalcanton, Julianne J, De Rosa, Alessandra, Duc, Pierre-Alain, Bianchi, Stefano, Capelo, Pedro R et al (2021) Old and new major mergers in the SOSIMPLE galaxy, NGC 7135. Monthly Notices of the Royal Astronomical Society, 502 (2). pp. 2296-2307. ISSN 0035-8711

It is advisable to refer to the publisher's version if you intend to cite from the work. http://dx.doi.org/10.1093/mnras/stab162

For more information about UCLan's research in this area go to http://www.uclan.ac.uk/researchgroups/ and search for < name of research Group>.

For information about Research generally at UCLan please go to http://www.uclan.ac.uk/research/

All outputs in CLoK are protected by Intellectual Property Rights law, including Copyright law. Copyright, IPR and Moral Rights for the works on this site are retained by the individual authors and/or other copyright owners. Terms and conditions for use of this material are defined in the policies page.

\section{CLoK}

Central Lancashire online Knowledge www.clok.uclan.ac.uk

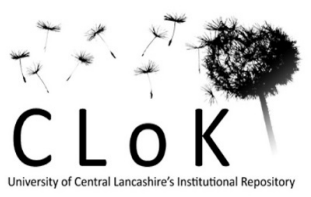




\title{
Old and new major mergers in the SOSIMPLE galaxy, NGC 7135
}

\author{
Thomas A. Davison ${ }^{\circledR}$, $1,2 \star$ Harald Kuntschner, ${ }^{1}$ Bernd Husemann ${ }^{\circledR}{ }^{\circledR}, 3$ Mark A. Norris ${ }^{\circledR}, 2 \star$ \\ Julianne J. Dalcanton, ${ }^{4}$ Alessandra De Rosa, ${ }^{5}$ Pierre-Alain Duc, ${ }^{6}$ Stefano Bianchi ${ }^{\circledR},{ }^{7}$ Pedro R. Capelo ${ }^{\circledR 8}$ \\ and Cristian Vignali ${ }^{(19,10}$ \\ ${ }^{1}$ European Southern Observatory, Karl-Schwarzschild-Strasse 2, D-87548 Garching bei Muenchen, Germany \\ ${ }^{2}$ Jeremiah Horrocks Institute, University of Central Lancashire, Preston PR1 2HE, UK \\ ${ }^{3}$ Max-Planck-Institut für Astronomie, K”onigstuhl 17, D-69117 Heidelberg, Germany \\ ${ }^{4}$ Department of Astronomy, University of Washington, Box 351580, Seattle, WA 98195, USA \\ ${ }^{5}$ INAF - Istituto di Astrofisica e Planetologie Spaziali, Via Fosso del Cavaliere, I-00133 Rome, Italy \\ ${ }^{6}$ Université de Strasbourg, CNRS, Observatoire astronomique de Strasbourg (ObAS), UMR 7550, F-67000 Strasbourg, France \\ ${ }^{7}$ Dipartimento di Matematica e Fisica, Università degli Studi Roma Tre, via della Vasca Navale 84, I-00146 Roma, Italy \\ ${ }^{8}$ Center for Theoretical Astrophysics and Cosmology, Institute for Computational Science, University of Zurich, Winterthurerstrasse 190, CH-8057 Zürich, \\ Switzerland \\ ${ }^{9}$ Dipartimento di Fisica e Astronomia, Alma Mater Studiorum, Università degli Studi di Bologna, Via Gobetti 93/2, I-40129 Bologna, Italy \\ ${ }^{10}$ INAF - Osservatorio di Astrofisica e Scienza dello Spazio di Bologna, Via Gobetti 93/3, I-40129 Bologna, Italy
}

Accepted 2021 January 17. Received 2021 January 16; in original form 2020 November 20

\begin{abstract}
The simultaneous advancement of high resolution integral field unit spectroscopy and robust full-spectral fitting codes now make it possible to examine spatially resolved kinematic, chemical composition, and star-formation history from nearby galaxies. We take new MUSE data from the snapshot optical spectroscopic imaging of mergers and pairs for legacy Exploration (SOSIMPLE) survey to examine NGC 7135. With counter-rotation of gas, disrupted kinematics and asymmetric chemical distribution, NGC 7135 is consistent with an ongoing merger. Though well hidden by the current merger, we are able to distinguish stars originating from an older merger, occurring 6-10 Gyr ago. We further find a gradient in ex-situ material with galactocentric radius, with the accreted fraction rising from 0 per cent in the galaxy centre, to $\sim 7$ per cent within 0.6 effective radii.
\end{abstract}

Key words: galaxies: evolution-galaxies: interactions-galaxies: stellar content.

\section{INTRODUCTION}

Galaxy merger research has shown how fundamental merging is to galaxy evolution, with historical merger rates generally increasing with galaxy mass (Bundy et al. 2009; Schawinski et al. 2010; L'Huillier, Combes \& Semelin 2012; Pillepich et al. 2018). Distant galaxies $(z \approx 2)$ are often quoted as being a factor of $2-5$ times smaller than those found locally (Daddi et al. 2005; Van Dokkum et al. 2008; Saracco, Longhetti \& Andreon 2009). As such it is widely assumed that a large amount of mass-assembly after $z \approx$ 2 is a result of hierarchical growth through galaxy mergers and accretion which has been widely corroborated from galaxy evolution models. Not only does merger history impact on almost all other aspects of galaxy evolution, but many galaxies have experienced large mergers throughout their history with around 50 per cent of galaxies experiencing a major merger (Maller et al. 2006), and essentially all surviving galaxies experiencing minor mergers, with frequency increasing with merger mass-ratio (Lotz et al. 2011). The exception for these cases are some rare pristine galaxy types $(\lesssim 0.1$ per cent of galaxies according to Quilis \& Trujillo 2013) which have likely

^E-mail: tdavison@uclan.ac.uk (TD); mnorris2@uclan.ac.uk (MN) experienced no outside interaction or accretion events (Trujillo et al. 2013).

Modelling is an excellent way to delve into the mechanics and subsequent effects of galaxy mergers. Using simulations, the exsitu mass fraction of accreted galaxies has been explored in depth (Pillepich, Madau \& Mayer 2015; Qu et al. 2017; Davison et al. 2020). This is useful for defining expected current merger rates to be compared to observationally. A challenging aspect of observational astronomy is demonstrating the merger history of observed nearby galaxies to verify these models, particularly if potential mergers occurred several Gyr ago.

Integral field spectroscopy has proven particularly useful in exploring galaxy kinematics and populations. Integral field units (IFU's) have provided spatially resolved maps of galaxies which can be used to diagnose population differences and kinematic effects as a result of mergers. This has been shown to be effective in numerous observational cases (see e.g. Guérou et al. 2016; Faifer et al. 2017; Ge et al. 2019).

The impact of mergers and merger history on galaxy evolution is an important aspect to understand. For one thing, mergers are known to drive gas towards the galaxy centre (Mihos \& Hernquist 1995), causing active galactic nucleus (AGN) activity and black hole growth, which in turn can shut down or suppress star formation in the galaxy at large (Cales \& Brotherton 2015; Choi et al. 2015). On the other hand, 


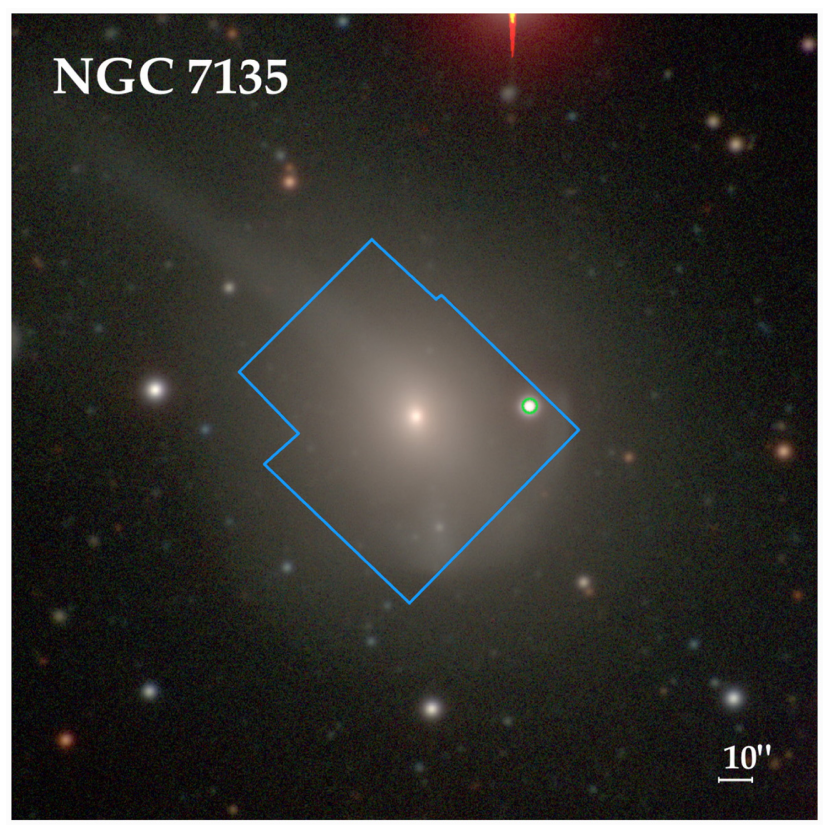

Figure 1. A colour image of NGC 7135 showing the MUSE cube footprint. Photometry of NGC 7135 is from the Carnegie-Irvine galaxy Survey (Ho et al. 2011). The blue border shows the boundaries of the reduced MUSE IFU data used in this study. A green circle traces an area containing a bright foreground star that was entirely excluded from the analysis.

mergers can cause sudden and significant bursts of star formation due to the disruption of previously unperturbed gas kinematics (Di Matteo et al. 2008; Ellison et al. 2013; Capelo et al. 2015; Moreno et al. 2015). Disruption in the gas kinematics of galaxies can leave key fingerprints in identification of merger events. One of the most readily identifiable features of a recent or ongoing merger is counter-rotating components, with up to 40 per cent of S0 galaxies displaying signatures of counter-rotation (Rubin 1994; Davis et al. 2011; Coccato et al. 2015; Bassett et al. 2017). Galaxy-galaxy mergers of the right combination can change the very morphological type of a galaxy. As such, mergers hold the power to define entire galaxy futures.

The S01-pec galaxy NGC 7135 (AM 2146-350, IC 5136) in the constellation of Piscis Austrinus is a merger remnant galaxy (Keel 1985) that is likely en route to forming an S0 galaxy. It currently displays several immediately striking visual features including an extended tail, shell features, and curved structure (Fig. 1) based on photometry from the Carnegie-Irvine galaxy Survey (Ho et al. 2011).

NGC 7135 was first described as having 'a curious jet and shell' in Malin \& Carter (1983) with the 'jet' later shown to be a tail in Rampazzo et al. (2003). The shell structures of the galaxy were found to be particularly clear in UV (Rampazzo et al. 2007; Marino et al. 2011), with FUV gas structure further linked to an accretion event that also likely formed the shells. Ueda et al. (2014) found $\mathrm{CO}$ emitting gas that was unassociated with the nucleus, along with $3 \mathrm{~mm}$ continuum associated with the nucleus. Despite speculation, NGC 7135 was determined to have no active nucleus as shown in Zaw, Farrar \& Greene (2009) through optical spectra analysis. Analysis in Keel (1985) identifies NGC 7135 as a merger galaxy, and in Rampazzo et al. (2003) NGC 7135 is shown to possess an elongated, asymmetric gas structure relative to the stellar material.

The local environment of NGC 7135 is described by Samir et al. (2016) as being 'low density', with the classification of 'low density'
(Annibali et al. 2010) a result of the richness parameter $\rho_{x y z}=$ $0.32 \mathrm{gal} \mathrm{Mpc}^{-3}$ (Tully \& Fisher 1988). Early-type galaxies in low density environments are known to possess on average younger populations ( $\sim 2$ Gyr younger) than similar galaxies in higher density environments (Thomas, Maraston \& Bender 2003), a likely result of more recent mergers and star formation.

In this work, we present new observations of the galaxy NGC 7135, recently obtained with MUSE. We aim to show that NGC 7135 is currently undergoing a major merger, with a history of older mergers underlying in the galaxy populations. The paper is presented as follows: In Section 2, we describe the motivation behind the observations, as well as the data reduction and limitations. In Section 3, we describe our methodology, including the use of regularization during spectral fitting. In Section 4, we present the resultant maps of stellar populations and kinematics, as well as gas properties similarly derived, including rotation differences between the two components. In Section 5, we discuss the implications of the results and finally in Section 6 , we provide a summary and concluding remarks.

\section{OBSERVATIONS AND DATA REDUCTION}

We observed NGC 7135 with the Multi-Unit Spectroscopic Explorer (MUSE; Bacon et al. 2010, 2014) at the Very Large Telescope (VLT) as part of the Snapshot Optical Spectroscopic Imaging of Mergers and Pairs for Legacy Exploration (SOSIMPLE) survey (Program ID: 0103.A-0637(A), PI: B. Husemann). The aim of the SOSIMPLE survey is to provide complementary IFU observations for an ongoing Hubble filler gap snapshot imaging program (Program ID: 15446, PI: J. Dalcanton). HST imaging of NGC 7135 is not yet taken due to the filler nature of the HST program, thus these MUSE observations act as a first look at the data, to which HST data can be compared to at a later date. Combining IFU spectroscopy with a large set of high-quality ancillary data will hopefully provide observational and theoretical insights into the evolution of merging systems.

The MUSE observations were conducted on 2019 July 6 during dark sky conditions and split into $3 \times 560$ s dithered pointings along with a $300 \mathrm{~s}$ dedicated blank sky field exposure for background subtraction of this extended galaxy. Rotations of $90^{\circ}$ were applied between exposures covering approximately $3.4 \mathrm{arcmin}^{2}$ as shown in Fig. 1. The seeing during the observations maintained at $\sim 1 \operatorname{arcsec}$, and the sky was covered with thin clouds during strong wind conditions from North-West direction.

The data were reduced with the standard ESO pipeline (Weilbacher et al. 2020) which performs detector calibrations, flat-fielding, wavelength calibration, flux calibration as well as sky subtraction, exposure alignment, and cube reconstruction of the combined exposures. We performed an additional correction for residual sky lines using a simple PCA algorithm. The MUSE pixel scale is $0.2 \operatorname{arcsec}$ pixel $^{-1}$, with a mean spectral resolution of $\sim 2.5 \AA$ though this can vary across the wavelength range (see fig. 5 of Husser et al. 2016). The resulting mean signal-to-noise ( $\mathrm{SN}$ ) ratio of the spaxels in the MUSE image within a wavelength range of 4759-6849 A (limited from 4759-9300 $\AA$ ) is 9.5, with a maximum spaxel SN of 131 .

\section{METHODOLOGY}

Spaxels were Voronoi binned to a minimum SN of 50 per $\AA$, thereby poor signal regions were made available for analysis, whilst higher SN spaxels remained unbinned. This optimally allowed for spatial investigation of spectral properties, without losing valuable high resolution data at high $\mathrm{SN}$ locations. 
The wavelength was restricted to 4759-6849 $\AA$ for all spaxels to ensure the strongest Balmer lines were included, and to exclude noisier sky-dominated regions at redder wavelengths. All spectra of spaxels within a bin were summed into a single spectra representing the area covered by the bin. An area containing a foreground star was masked from analysis in the West of the image (see Fig. 1).

To analyse the spectra from the binned NGC 7135 data, we utilized the Penalized PiXel-Fitting (pPXF) method, described in Cappellari \& Emsellem (2004) and upgraded in Cappellari (2017). With this method, single-age single-metallicity stellar population (SSP) models are fit to spectra to build a map of stellar populations across age and metallicity space. By identifying the combination of SSP models that approximate a given spectrum, the estimated constituent populations are extracted, as well as velocity and dispersion. Stellar models are weighted as per the estimated fraction of the population present in the galaxy. As a result, output weights of stellar models indicate the fractions of specific stellar populations present in the spectrum. The output model of combined spectra is made more physical by the use of template regularization (see e.g. section 3.5 of Cappellari 2017), the methodology of which is explained in detail below. Standard pPXF cleaning algorithms were included to mask emission lines where necessary.

A total of 552 MILES SSP models (Vazdekis et al. 2010) were used to fit to galaxy spectra. These models were of Kroupa revised initial mass function (log slope of $1.3, M_{\max }=100 \mathrm{M}_{\odot}$ ) using BaSTI isochrones, with a metallicity range of -2.27 to $+0.4[\mathrm{M} / \mathrm{H}]$ in 12 non-linear steps, and an age range of 0.1 to $14.0 \mathrm{Gyr}$ in 46 non-linear steps (Kroupa 2001; Cassisi et al. 2006; Pietrinferni et al. 2006; Falcón-Barroso et al. 2011; Vazdekis et al. 2012).

Application of regularization allows smoothing over stellar model weights to reproduce a population map consistent with physical results. The weighted templates that have been combined to produce a target spectrum will often be unphysically localized to only the strongest of possible solutions, with many other valid solutions being overlooked, despite their physicality. To produce more representative distributions, regularization seeks to smooth the solutions to a physical state. The challenge is to smooth the template weights to a solution that most accurately represents observed conditions, whilst not overlooking genuine fluctuations and details present in the model fit. The regularization parameter controls the strength of the smoothing and is deduced through a robust iterative approach for each spectrum individually. The regularization parameter is derived such that it corresponds to the maximum value consistent with observations. Thus the derived star formation history will be the smoothest that is consistent with the observations. This has been shown in literature to be an accurate and useful method of galaxy population extraction (see e.g. Comerón et al. 2015; Norris et al. 2015; Guérou et al. 2016; Faifer et al. 2017; Ge et al. 2019; Boecker et al. 2020).

In this work an iterative routine is applied to extract the optimal regularization parameter. For the best possible fit, the $\chi^{2}$ of the solution is expected to be approximately equal to the number of available voxels in the spectrum, $N$ (i.e. the number of voxels available after any masking). To obtain this optimal solution, the $\chi^{2}$ must be increased from the unregularized $\chi^{2}$ (referred to as $\chi_{0}^{2}$ ) by $\sqrt{2 N}$.

After rescaling noise from the unregularized solution such that $\frac{\chi^{2}}{N}=1$, we make a number of primary guesses at the regularization parameter. We find the $\Delta \chi^{2}$ of these initial guesses and fit a function to the input regularization guesses and output $\Delta \chi^{2}$ values. By doing so we can precisely find the optimal regularization parameter such that $\chi^{2}=\chi_{0}^{2}+\sqrt{2 N}$. This action is performed for every bin, resulting in optimal solutions across the entire image map.

\section{RESULTS}

We separate the analysis of NGC 7135 into three components; the stellar component analysis, encompassing the stellar kinematics; the gaseous component analysis, encompassing gas kinematics, emission lines and star formation aspects; and the population analysis, examining the various stellar populations and the resulting implications for the assembly history of NGC 7135.

To examine the stellar component, we utilize Voronoi binning as described in Section 3. From this we are able to examine the stellar rotation and bulk velocities, as well as mean age and metallicities spatially across the galaxy (Fig. 2). To investigate details related to the gaseous component, we use regular binning to view the gas velocities and rotation, as well as the line strengths of $\mathrm{H} \alpha$ and $\mathrm{H} \beta$ (Fig. 3). Though we see reasonable amounts of $\mathrm{H} \alpha$ emission, there is scant evidence for significant ongoing star formation. This is explained in detail in Section 4.2. Finally, in Section 4.3 we further analyse age and metallicity distributions for sampled regions across the galaxy to diagnose assembly history and current merger status, then go on to examine underlying metal-poor populations in Section 4.4.

\subsection{Stellar properties}

Application of the pPXF method to the NGC 7135 data cube provides mean kinematic properties which are extracted from each bin. Demonstrations of this for velocity and velocity dispersion of the galaxy are found in the top panels of Fig. 2. Application of regularization and mass-to-light ratios produce maps of the constituent stellar populations within each bin of the galaxy. From these bins, we can derive mean mass-weighted stellar age and metallicity values, as demonstrated in the lower panels of Fig. 2.

The stellar kinematic, age, and metallicity maps of NGC 7135 reveal much about the galaxy. Stellar rotation is immediately visible. This is of key interest when comparing to gas which rotates counter to the direction of stellar rotation. This is explored in detail in Section 4.2. One prominent kinematic feature, perhaps most clearly seen in the velocity map (top left-hand panel) of Fig. 2, is an arc of incongruous material at higher than average velocity, stretching from the south-west of the figure to the West. The southern end of this arc is matched in the metallicity map (lower right-hand panel, Fig. 2) by a higher metallicity region, which is also distinct in velocity and velocity dispersion. Upon inspection, this is revealed to be an infalling galaxy currently merging on to NGC 7135. This can be clearly seen in photometry, and even more compelling evidence comes from population analysis below.

\subsection{Gas properties}

To explore gas kinematics and distribution in NGC 7135, regular binning was employed to avoid biases caused by the stellar light controlling Voronoi binning. Large square bins containing 64 pixels were selected across the face of the data cube, and spectra within a given bin were summed and analysed with ppxf as described in Section 3. Following this, those bins with signal-to-noise that exceeded the minimum detection threshold were re-binned to a higher resolution. This adaptive 'zoom' binning gave high resolution in areas of strong $\mathrm{H} \alpha$ emission. The zoom resolution was limited to central regions of the galaxy, where the finest detail was required. 

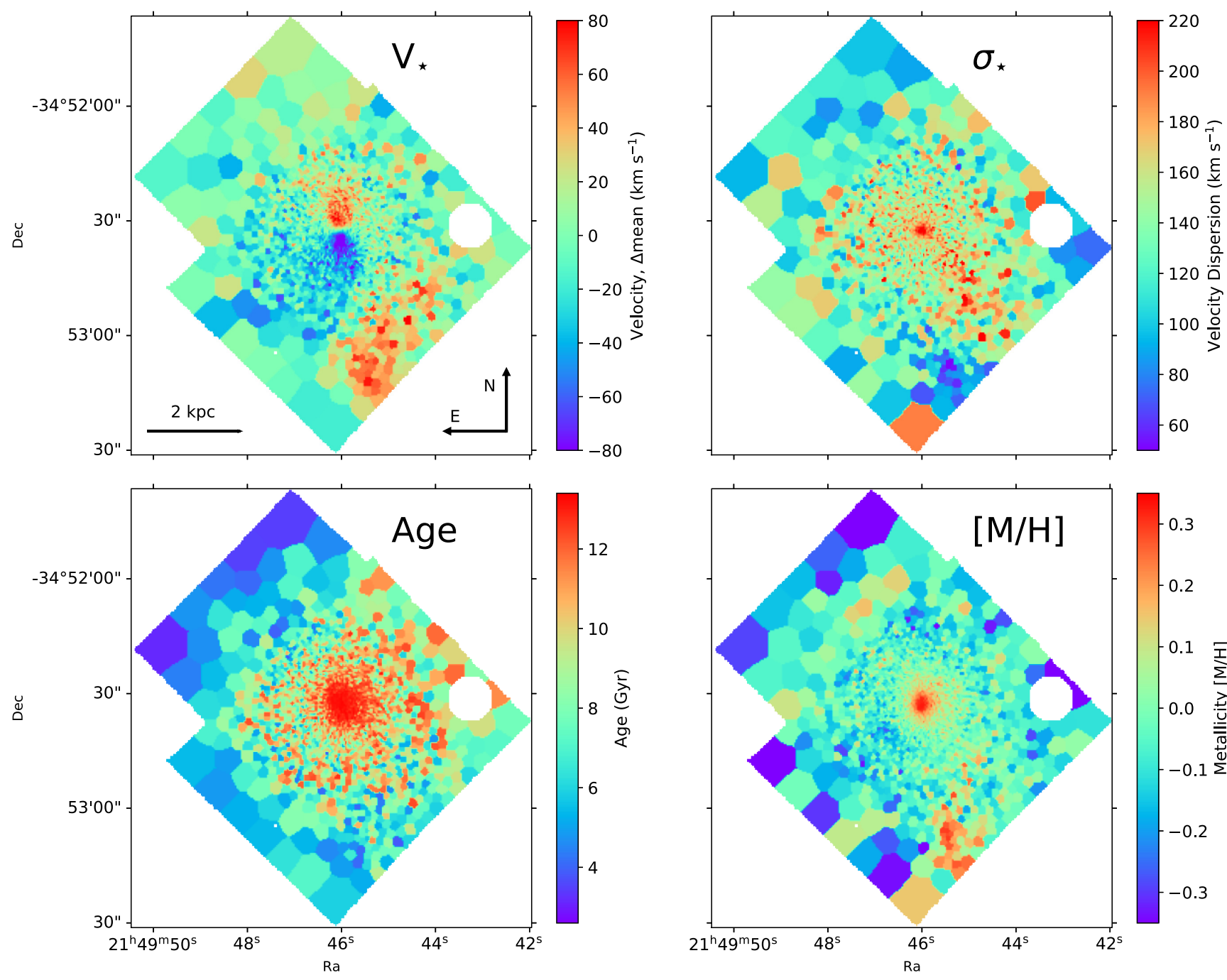

Figure 2. Voronoi map of NGC 7135 showing four different stellar kinematic or mass-weighted population properties. The top left-hand panel shows the mean velocity in $\mathrm{km} \mathrm{s}^{-1}$ for each bin. The top right-hand panel shows mean velocity dispersion within bins in $\mathrm{km} \mathrm{s}^{-1}$. The lower left-hand panel shows the mean age of populations within the bin in Gyr. Finally the lower right-hand panel shows mean metallicity within each bin. North is to the top of the image, and East is to the left. The stars show clear rotation in the centre. Velocity dispersion, age, and metallicity all increase towards the galaxy centre. Distinct kinematics and metallicity south of the centre highlight a distinct component.

NGC 7135 displays localized areas of strong Balmer emission, shown in Fig. 3 with a cropped version showing the galaxy centre in Fig. 4. As seen from all panels, the gas is asymmetric in distribution as well as in kinematics. The rotation of the gas highlights the decoupled nature of the stellar material in the core.

Gas is counter-rotating to the stellar component, strongly indicating a disrupted system. A slight deviation to the coherent gas movement is seen in the galaxy centre, giving an ' $S$ ' shaped gas rotation profile. Counter-rotation has long been associated with galaxy mergers (see e.g. Bertola, Buson \& Zeilinger 1988). Total decoupling of gas rotation from stellar components as a result of prograde-prograde merger shocks has been shown in simulation in Capelo \& Dotti (2017), and a similar event appears to be in play here, wherein a major merger has resulted in a counter-rotation of the gas component. Plausibly this is the result of a previous merger providing counter-rotation from a prograde-prograde merger, this is expanded further in Section 4.3. Alternatively, counter-rotation could have arisen as a result of a first pass of the currently infalling galaxy.
Velocity vectorization of the gas and stars allows us to measure the gas and stellar rotation misalignment. The rotation consensus in the gas is fairly standard, with the gas rotating around the centre. In the stellar component however, matters are complicated by the velocity of the in-falling galaxy, which shifts the positive rotation vector compared to the core. If we consider only the core, the misalignment of gas and stars is $176^{\circ}$, whereas when the entire cube is considered, the misalignment is $139^{\circ}$. This is entirely within the realm of expected values for an interacting galaxy (see e.g. Barrera-Ballesteros et al. 2015; Bryant et al. 2019). This is shown in Fig. 4 as solid and dashed arrows for the directions of mean positive stellar and gas rotation, respectively, with associated errors shown as shaded regions.

Regions of $\mathrm{H} \alpha$ emission can be seen in the southern areas of the lower left-hand panel of Fig. 3. This forms a large arc with patches exhibiting particularly strong emission. These are seemingly matched by arcs in the north in an asymmetrical manner.

Considering the gas asymmetry and the increase in both gas velocity and velocity dispersion, a large amount of gas can be 

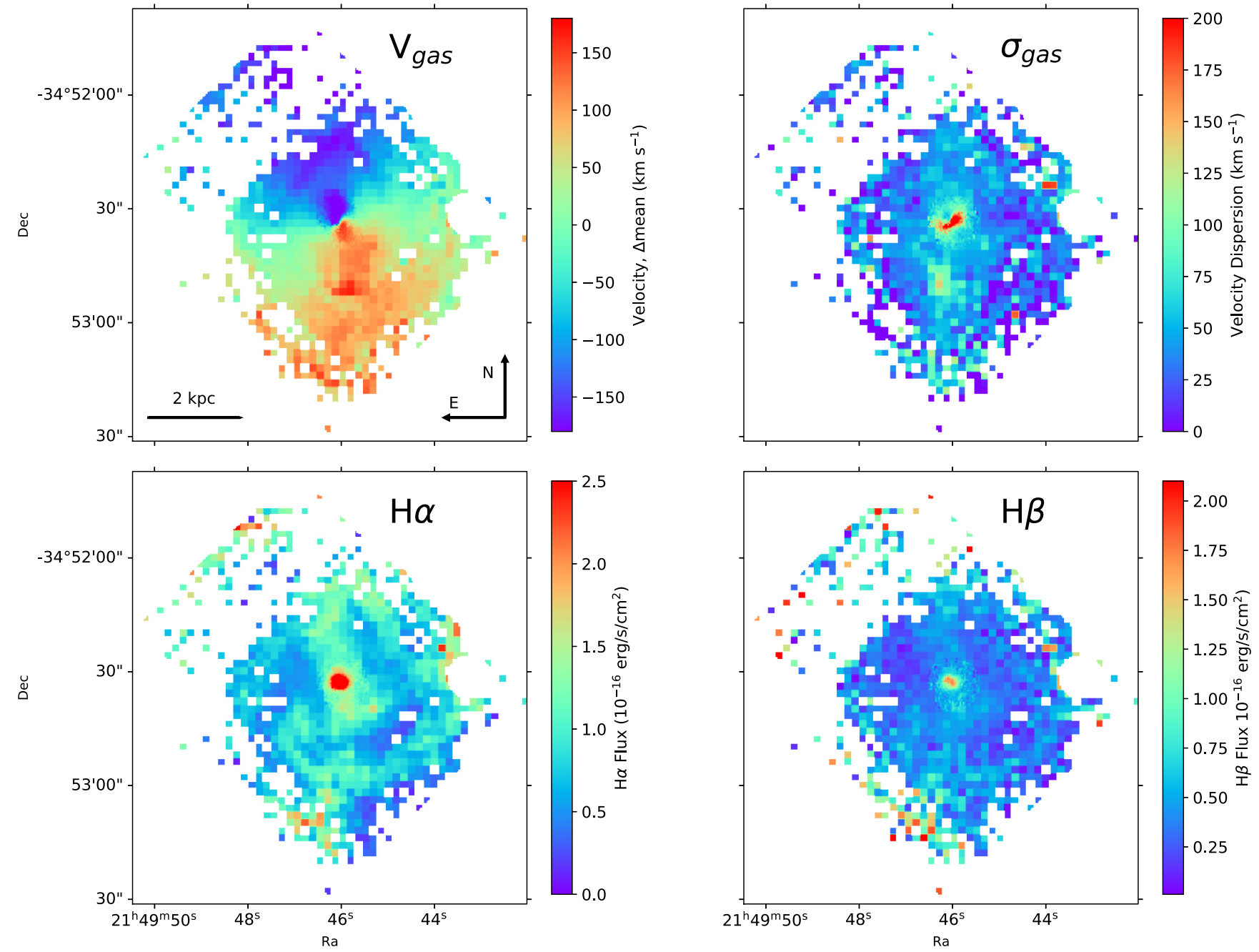

Figure 3. Regularly binned map of NGC 7135 showing four different gas kinematic and strength properties. The top left-hand panel shows the mean velocity of gas in $\mathrm{km} \mathrm{s}^{-1}$ for each bin. The top right-hand panel shows mean velocity dispersion of gas within bins in $\mathrm{km} \mathrm{s}^{-1}$. The lower left-hand panel shows the $\mathrm{H} \alpha$ flux throughout NGC 7135. The scale has been limited from the true maximum to better display regions of intermediate strength. This limits the core from a true strength of at most $36.2 \times 10^{-16} \mathrm{erg} \mathrm{s} \mathrm{cm}^{-2}$ (limited to $2.5 \times 10^{-16} \mathrm{erg} \mathrm{s} \mathrm{cm}^{-2}$ ). The lower right-hand panel shows $\mathrm{H} \beta$ flux throughout NGC 7135 . The scale has been limited from the true maximum to better display regions of intermediate strength. This limits the core from a true strength of at most $5 \times 10^{-16} \mathrm{erg} \mathrm{s}^{-2}$ (limited to $2.1 \times 10^{-16} \mathrm{erg} \mathrm{s} \mathrm{cm}^{-2}$ ). The gas velocity shows counter-rotation compared to the stellar component, and on a slightly different axis, suggesting a merger origin.

attributed to material stripped from the outskirts of the infalling galaxy and which is currently in the process of accreting on to the host galaxy. This is seen in the largest area of gas velocity dispersion occurring outside the core, located in a tight region south of the galaxy core. This region indicates a quantity of gas that is not associated with the cohort gas of NGC 7135, as it displays a region where infalling gas is interacting with the galaxy interstellar medium. This area of higher than expected dispersion is in the plane of the galaxy gas rotation, again evidence that gas is infalling, creating high velocity dispersion at the position where in-situ gas meets ex-situ gas.

A strong presence of $\mathrm{H} \alpha$ in concentrated regions is consistent with the picture of NGC 7135 as a galaxy that has perhaps recently undergone star formation as suggested in Rampazzo et al. (2007), though at low levels. Despite this, there is little to no evidence of strong ongoing star formation. This can be seen in the emission-line diagnostic diagram in Fig. 5. Almost all the sources of emission are associated with low-ionization nuclear emission-line regions (LINERs). Though a handful of AGNs sources can be seen, they largely lie in the outer noisier regions of the data cube, which makes the presence of true AGN sources doubtful, as shown in Zaw et al. (2009). This strong bias towards LINER emission is typical of merging systems with shock-driven LINER emission (Monreal-Ibero et al. 2010; Rich, Kewley \& Dopita 2011).

ALMA data (Ueda et al. 2014) showing the ${ }^{12} \mathrm{CO}(J=1-$ 0 ) emission is overlaid in the lower left-hand panel of Fig. 4. The ALMA observations reveal a significant peak in CO emission offset from the galaxy core with an integrated molecular gas mass of $M_{\mathrm{H} 2}=(5.4 \pm 1.4) \times 10^{7} \mathrm{M}_{\odot}$ adopting an $\alpha_{\mathrm{CO}}=$ $4.8 \mathrm{M}_{\odot} \mathrm{pc}^{-2}\left(\mathrm{~K} \mathrm{~km} \mathrm{~s}^{-1}\right)^{-1}$ (Solomon \& Barrett 1991). This cold gas mass would correspond to an expected SFR of only $\sim 0.025 \mathrm{M}_{\odot} \mathrm{yr}^{-1}$ if a normal depletion time of $2 \mathrm{Gyr}$ for galaxies is assumed (Bigiel et al. 2011; Leroy et al. 2013). Although there is no similarly distinct ionized gas structure observed with MUSE, there is plenty of ionized gas which may partially originate from star formation despite the LINER-like classification. The extinction-corrected $\mathrm{H} \alpha$ flux within the central $r=1 \operatorname{arcsec}$ is $(4 \pm 0.4) \times 10^{-13} \mathrm{erg} \mathrm{s}^{-1} \mathrm{~cm}^{-2}$ 

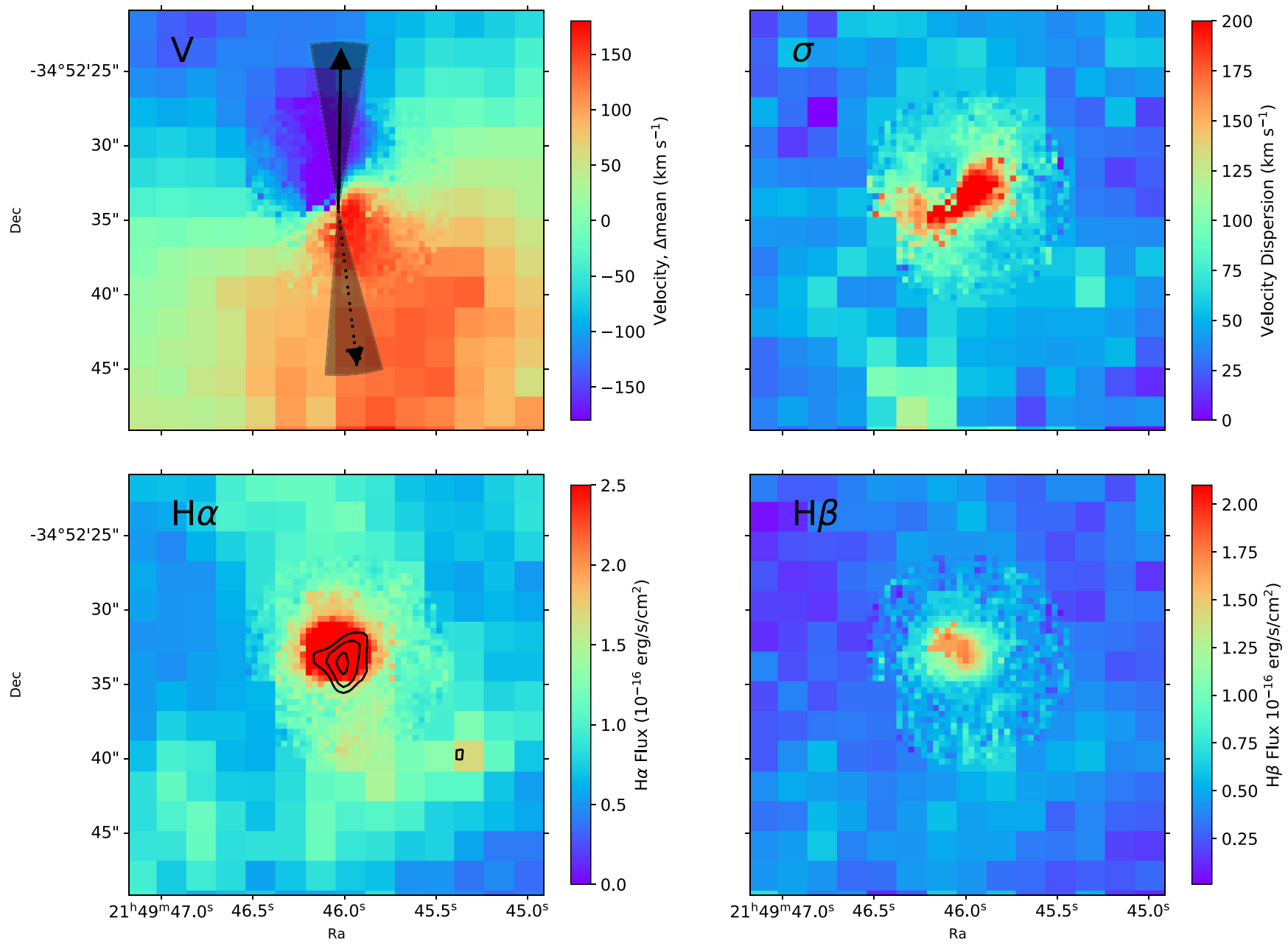

Figure 4. Regularly binned and zoomed in map of NGC 7135 showing four different gas kinematic and strength properties. The top left-hand panel shows the mean velocity of gas in $\mathrm{km} \mathrm{s}^{-1}$ for each bin. The top right-hand panel shows mean velocity dispersion of gas within bins in $\mathrm{km} \mathrm{s}^{-1}$. The lower left shows the $\mathrm{H} \alpha$ flux throughout NGC 7135. The scale has been limited from the true maximum to better display regions of intermediate strength. This limits the strongest emission near the core from a true strength of at most $36.2 \times 10^{-16} \mathrm{erg} \mathrm{s} \mathrm{cm}^{-2}$ (limited to $2.5 \times 10^{-16} \mathrm{erg} \mathrm{s} \mathrm{cm}^{-2}$ ). The lower right-hand panel shows $\mathrm{H} \beta$ flux throughout NGC 7135. The scale here has also been limited. This limits the strongest emission from a true strength of at most $5 \times 10^{-16} \mathrm{erg} \mathrm{s} \mathrm{cm}^{-2}($ limited to $2.1 \times 10^{-16} \mathrm{erg} \mathrm{s} \mathrm{cm}^{-2}$ ). In the upper left-hand panel, arrows show the average positive rotation direction. The solid arrow indicates the average stellar component positive rotation whilst the dotted arrow shows the average gas positive rotation direction. Shaded regions show the standard deviation of vectors for both components for bins of 0.1 effective radii. In the lower left-hand panel, contours show integrated $\operatorname{CO}(J=1-0)$ emission detected in ALMA observations (Ueda et al. 2014). Contours show the $0.8,1.0$, and $1.2 \mathrm{Jy} \mathrm{km} \mathrm{s}^{-1}$ levels. There is pervasive $\mathrm{H} \alpha$ emission with a high luminosity and high velocity dispersion component in the centre, though there is little evidence of star formation.

which would correspond to $\mathrm{SFR}=0.5 \pm 0.05 \mathrm{M}_{\odot} \mathrm{yr}^{-1}$ following Kennicutt Jr (1998). So only 5 per cent of the central $\mathrm{H} \alpha$ would need to be hidden among LINER-like classified ionized gas to be in agreement with ongoing star formation. Such a low fraction of star formation would not alter the line diagnostics significantly and would remain hidden. Hence, we cannot rule out ongoing star formation based on the central cold gas mass observed by Ueda et al. (2014). Given the highly disturbed kinematics, the possibility that dynamical suppression of star formation is preventing cold gas collapse cannot be tested by our observations.

\subsection{Stellar population mapping}

Populations of a galaxy evolve in metallicity over time, gradually enriching with age. The exact quantities and rates of this enrichment are well known (Carraro \& Chiosi 1994; Layden \& Sarajedini 2000; Pont \& Eyer 2004), with the rate of enrichment directly tied to galaxy mass resulting in the mass-metallicity relation. Thus, we can quickly establish whether a galaxy has followed the standard enrichment of its population as would be expected from an isolated galaxy.

In reality, galaxies are more often than not experiencing regular disturbances in the form of mergers, fly-bys, and intracluster medium interaction such as ram-pressure stripping (Lotz et al. 2011; Sinha \& Holley-Bockelmann 2012; Ebeling, Stephenson \& Edge 2014; Ventou et al. 2017). One effect of this is the variation of the agemetallicity relation of a galaxy from the modelled form. This is most strikingly clear when a galaxy accretes material from a lower mass galaxy (Spolaor et al. 2009; Leaman, VandenBerg \& Mendel 2013). Due to the lower metal enrichment rate of lower mass galaxies than that of larger mass galaxies, one finds that in general a smaller mass galaxy will exhibit far lower values of metallicity at late ages. Because of the ability for full spectral fitting methods to identify populations based on age and metallicity models, one would see these two populations as distinct and separate areas on an age- 


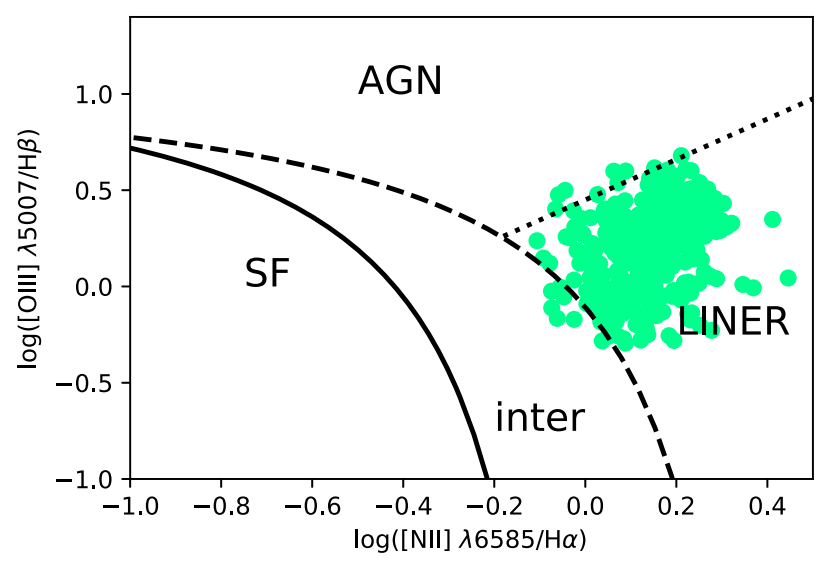

Figure 5. An emission-line diagnostic diagram (Baldwin, Phillips \& Terlevich 1981) divided into various sources. Each bin is shown as a point according to its emission ratios of $[\mathrm{N} \mathrm{II}] / \mathrm{H} \alpha$ and $[\mathrm{O} \mathrm{III]}] / \mathrm{H} \beta$ allowing for the identification of regions of star formation, AGN emission, or Low-ionization nuclear emission-line region (LINER) emission. Detailed description of the line equations can be found in Park, Sohn \& Sukyoung (2013). NGC 7135 shows no bins where current star formation is clear in the emission. Slight overlaps outside the LINER emission bin are unlikely to be genuine, but rather likely arise because of noise and intrinsic variations. The galaxy emission is overwhelmingly LINER type.

metallicity diagram. This is dependent on the difference in mass of the mergers however, as if two galaxies of similar mass were to merge, the separation of populations on the age-metallicity diagram would be too little to distinguish at the current resolutions of fullspectral fitting methods. Using these principles, we can estimate which of the populations present are those which have accreted on to the host galaxy, and are therefore ex-situ in origin.

We apply these principles to the population maps of NGC 7135 in order to derive the history of formation and evolution. In Fig. 6, nine regions are marked with sequential letters corresponding to population maps, which are similarly sequentially lettered, with maps taken from the Voronoi bin below the labelled cross. Each position marks an area of interest or standard uniformity across the maps of Fig. 2 with which we can build a picture of the assembly and current status of NGC 7135. Region 'A' marks the core of NGC 7135. Regions ' $\mathrm{B}$ ' and ' $\mathrm{C}$ ' sample the tidal tail clearly seen in the unsharp mask image (lower right-hand panel of Fig. 6), with increasing galactocentric radius. Regions 'D', 'E', and 'F' also sample with increasing galactocentric radius, however they do so outside of any prominent tidal features. These are assumed to be a 'control' sample which are chosen to represent the underlying galaxy, though show signs of probing accreted material. Regions ' $G$ ' and ' $H$ ' sample the tidal regions opposite the tail, with ' $\mathrm{H}$ ' particularly covering unstripped remnants of the infalling galaxy. Finally region ' $K$ ' covers the core of the infalling galaxy.

Starting with region 'A', we see a very high metallicity, very old population associated with the galaxy core. This is to be expected and is commonly seen in galaxy cores (see e.g. Guérou et al. 2016). There is little obvious evidence for accreted populations as expected, as shown by the old and high metallicity population, and lack of any clear population bi-modality.

Moving along the main tidal tail in region ' $\mathrm{B}$ ', we see a much younger population at high metallicity. When comparing to regions not associated with tidal features but at similar radius such as ' $E$ ' and ' $F$ ', we see that the population of ' $B$ ' is not comparable to ' $E$ ' or ' $F$ '. This is largely due to a lack of older material that would be expected to be associated with the host galaxy. Plausibly this is the result of the vast majority of the stellar material originating in the infalling galaxy and comprising the tidal tail, and thus the populations visible are instead associated with this infalling object, rather than original populations of NGC 7135. A small amount of material is also visible as a young and metal-poor population. This can be attributed to ex-situ material that merged on to either NGC 7135 or the infalling galaxy in the past prior to the current merger, and thus shows a separate population signature.

As we move further out along the tidal tail to region ' $\mathrm{C}$ ', many of the features become more prominent. For one thing, the high metallicity population associated with the stripped material from the infalling galaxy remains. Furthermore, low metallicity ex-situ populations increase in the fraction of contributed mass (as seen as a distinctly separate low metallicity population). Care must be taken in comparison due to colour normalization differences on the plot, however the maximum low metallicity ex-situ fraction increases from $\sim 0.5$ per cent in ' $\mathrm{B}$ ' to $\sim 1.0$ per cent in ' $\mathrm{C}$ ', with a higher sum total of ex-situ material. This increase is to be expected, as ex-situ material commonly increases in fraction with galactocentric radius (La Barbera et al. 2012; Martin et al. 2018; Davison et al. 2020). It is unclear whether this ex-situ population is associated with NGC 7135 or the infalling galaxy, however it could plausibly be from both, as models of hierarchical growth suggest both galaxies would have undergone historical minor mergers in all but the rarest cases (Fakhouri, Ma \& Boylan-Kolchin 2010). A burst of star formation is also seen in the final Gyr history. This is suggestive of a rapid star formation event, most likely triggered as a result of the galaxy interactions. Following this, no star formation is noticed in any bin. A shutdown of star formation after a major merger is discussed widely in literature (see e.g. Bekki 2001; Barr et al. 2007; Cortesi et al. 2013; Querejeta et al. 2015; Puglisi et al. 2021).

Region 'D' samples an inner region of NGC 7135. It shows similar populations as in 'A', however extends slightly to lower ages as expected following galaxy population age gradients. Little to no ex-situ material is clear. Moving further out in radius, we come to region ' $\mathrm{E}$ '. This also shows the expected populations previously seen in ' $A$ ' and ' $D$ '. This time however there is a more significant low metallicity ex-situ population, which as mentioned previously is expected as one reaches regions further from the galaxy centre according to galaxy simulations. Also prominent in region ' $E$ ' is a population of intermediate age and high metallicity stars. As shown below in region ' $\mathrm{H}$ ', this is almost certainly associated with the infalling galaxy.

Region ' $F$ ' samples at a slightly greater radius than ' $E$ ', again with more prominent features, though in similar positions to 'E'. We see an increase in the low metallicity ex-situ population radially along the tidal tail ('A', 'B', and ' $\mathrm{C}$ ') and well as radially in areas not associated with tidal features ('D', 'E', and 'F').

The final regions sample the galaxy shell and associated infalling object. Region 'G' examines an area of tidal shell seemingly also originating from the infalling galaxy. The region almost identically matches ' $\mathrm{H}$ ' which is placed to examine the outskirts of the infalling object, in regions that have yet to be stripped. The fact that these two populations are quite so similar suggests they are of the same origin, and that the tidal shells and tails are the result of scattered accreted material from the infalling galaxy.

Finally region ' $\mathrm{K}$ ' examines the core of the infalling galaxy at approximately 0.5 effective radii from the centre of NGC 7135 . It shows a highly metal-rich and old population with the exact tendencies of a galaxy nucleus. It shows largely the same properties as the nucleus of NGC 7135, though with marginally 

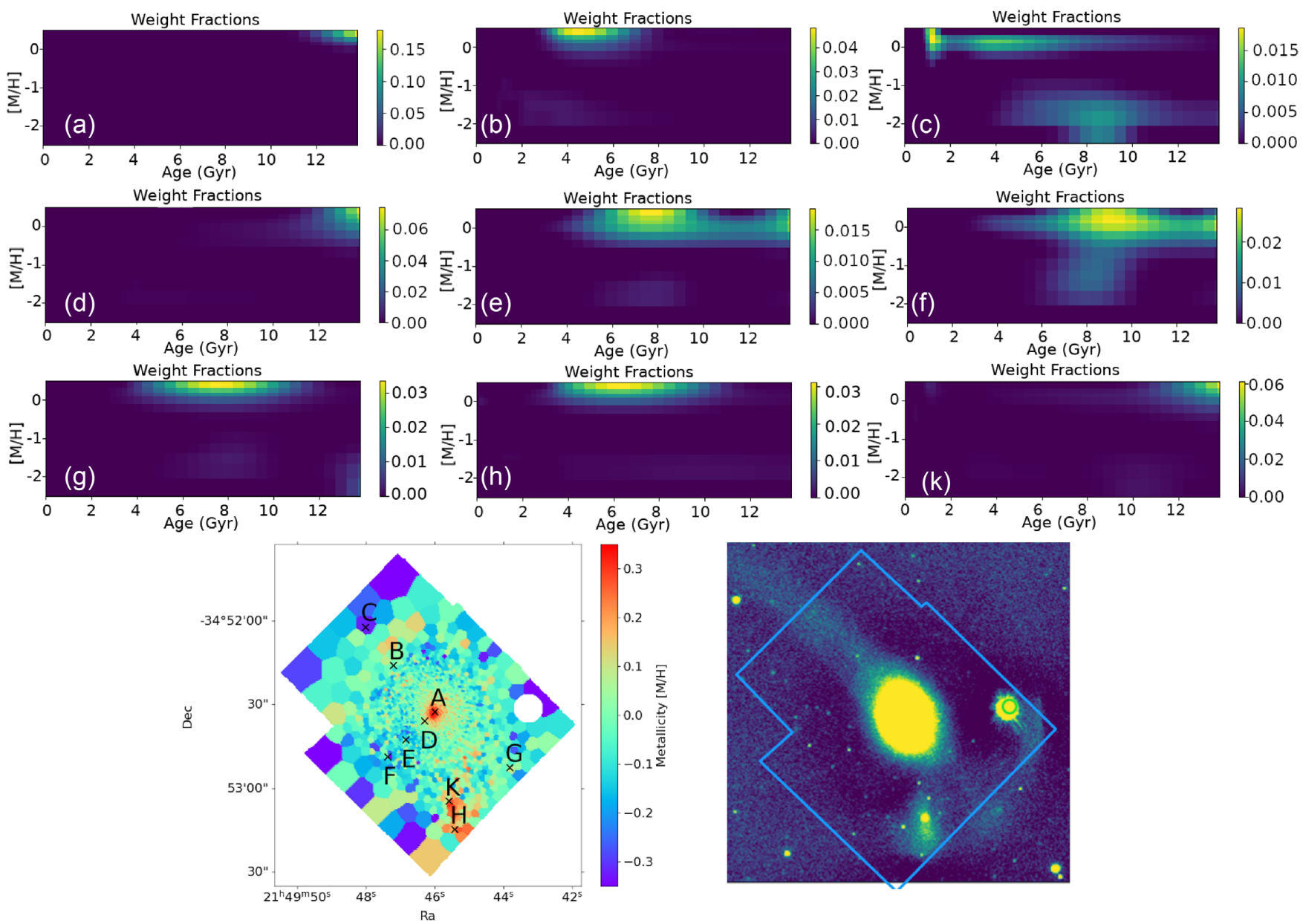

Figure 6. NGC 7135 population sampling diagram. The upper nine panels display mass-weighted metallicity space of NGC7135 for various regions. Corresponding regions are marked in the lower left-hand panel with crosses marking the position extracted, and the corresponding letter. The lower righthand panel shows the same region as an unsharp masked image to highlight tidal features. Data for the unsharp masked image are taken from the VST ATLAS survey (Shanks et al. 2015). The diagrams build a narrative in which a recent and ongoing merger creates large tidal features in NGC 7135. There are also populations of far lower metallicity which are well mixed in the galaxy. These populations indicate historical mergers of high merger-mass ratio.

lower metallicity and a greater extent in age, suggesting a lower mass.

The velocity dispersion of region ' $K$ ' (seen in Fig. 2) is at a much lower average velocity dispersion than the host galaxy, again suggesting a lower mass of the merging galaxy compared to NGC 7135. This is curious considering its high metallicity. One explanation would be that the in-falling galaxy is the remnant of a galaxy core stripped of its halo, which would explain both its relatively high brightness and high metallicity. This is also supported by the large amounts of seemingly ex-situ gas that are seen in Fig. 3, where this gas would have formed the outer regions of the infalling galaxy as explained further in Section 4.2.

The velocity dispersion (Fig. 2) increases significantly midway between the accreting galaxy core and the host galaxy core. This further lends weight to the idea that material is accreting on to the host galaxy, as the high velocity dispersion area indicates a region where accreted material begins encountering large amounts of insitu material, and the difference in velocities becomes more evident, inflating the velocity dispersion, prior to mixing.

In summary, the population maps are indicative of three distinct galaxy populations, in which two significant merger events are present. The first is ongoing, with an intact core of a second galaxy currently in close proximity to NGC 7135, with material being stripped off, accreted on to NGC 7135, and creating large tidal features. These make up the high metallicity populations at intermediate ages. Yet another population is consistently present, as a low metallicity, intermediate to old aged population. As discussed previously, chemical enrichment and mass-metallicity relations mean this population is not associated with either galaxy. Therefore, we attribute these stars to older historical mergers, now mixed loosely with the main populations. It is unclear which of these two present galaxies these populations accreted to, however as mentioned previously, the ex-situ population is likely present in both galaxies independently, and was captured by each prior to this ongoing merger.

\subsection{Accreted populations}

As seen in Fig. 6, many bins display a bi-modality in population distribution (see e.g. panels 'B', 'C', 'E', 'F', 'G', and 'H'). Such a strong separation in populations suggests stellar material being obtained from more than a single source. Galaxies not associated with the main galaxy will evolve with a different metallicity due to the mass-metallicity relation. As such, when the galaxies merge, there will be a distinct separation in the age-metallicity relation of each 


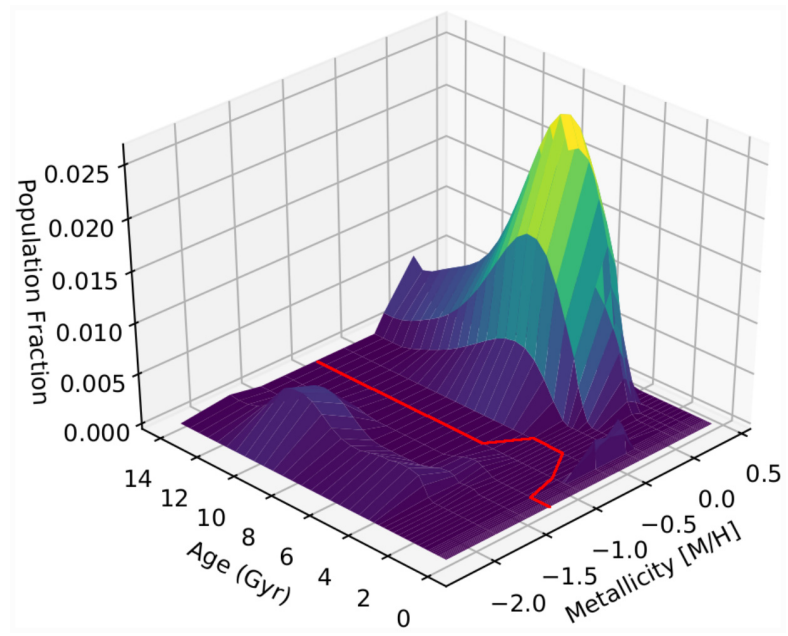

Figure 7. Population map of one bin projected on 3D axes. A line is sought for each map to bisect the lower metallicity population from the older using low saddle points. For this example, the path is marked by a red line.

galaxy. The most obvious explanation for the bimodal populations seen in Fig. 6 would be the merger of a less massive, lower metallicity galaxy to the host galaxy or on to the infalling galaxy, beginning 10 Gyr ago. Furthermore, the fact that the bi-modality of populations is seen at almost all positions across the galaxy outside of the cores (panels 'B', 'C', 'E', 'F', 'G', and 'H') suggests that this material has been well mixed and is distributed throughout the galaxy, with the exception of the two galaxy cores (see panels 'A', 'D', and ' $\mathrm{K}$ ').

To explore the population bi-modality, the fraction of stars not associated with the main host population was determined from each bin. To identify two discontinuous populations, a dividing line was sought across the population map, which would follow the lowest saddle points. This 'path of least resistance' then divided the populations into two distinct sources; one being material from NGC 7135 and the in-situ material of the infalling galaxy; and the other source being low metallicity populations accreted on to both galaxies at earlier times. This can be imagined as the valley between two hills, with the dividing line taking the natural path of a river at the lowest potential. This is visualized in Fig. 7 with a red line showing the calculated separation path for one random bin, separating the populations into two sources.

Application of this to all bins provides a map such as in Fig. 8, where we can examine the fraction of stellar material associated with the lower metallicity source. Fig. 8 shows a polar view of NGC 7135 to better examine radial features. By examining fraction across the galaxy, we can infer regions of higher or lower concentration of the accreted material.

At the centre of NGC 7135, we see no accreted material suggesting the core is dominated by in-situ stars. The density of accreted material rises with radius which is indicative of galaxy mergers depositing material on the outer regions of the galaxy. The material seems to be unevenly radially mixed, with proportionally higher quantities of ex-situ material deposited between 0 and 1 radians from North. This is likely a projection effect, as the area at the south of the galaxy (the left-hand and right-hand extents of Fig. 8) aligns with the previously mentioned high metallicity galaxy, with the stream of stellar material obscuring the host galaxy structure, and dominating the spectral light.

We can further see evidence of the division of the various populations by examining stellar mass estimates per population, determined with the division of the age-metallicity plane in combination with mass-to-light ratios. We show this in Fig. 9, with three regions of different populations separated roughly. Using mass-tolight ratios from Thomas et al. (2003), we estimate the stellar mass per population division, per pixel. The panel labelled ' 1 ' corresponds to intermediate age stars with high metallicity which were associated with the infalling galaxy. This is confirmed in the first map in the

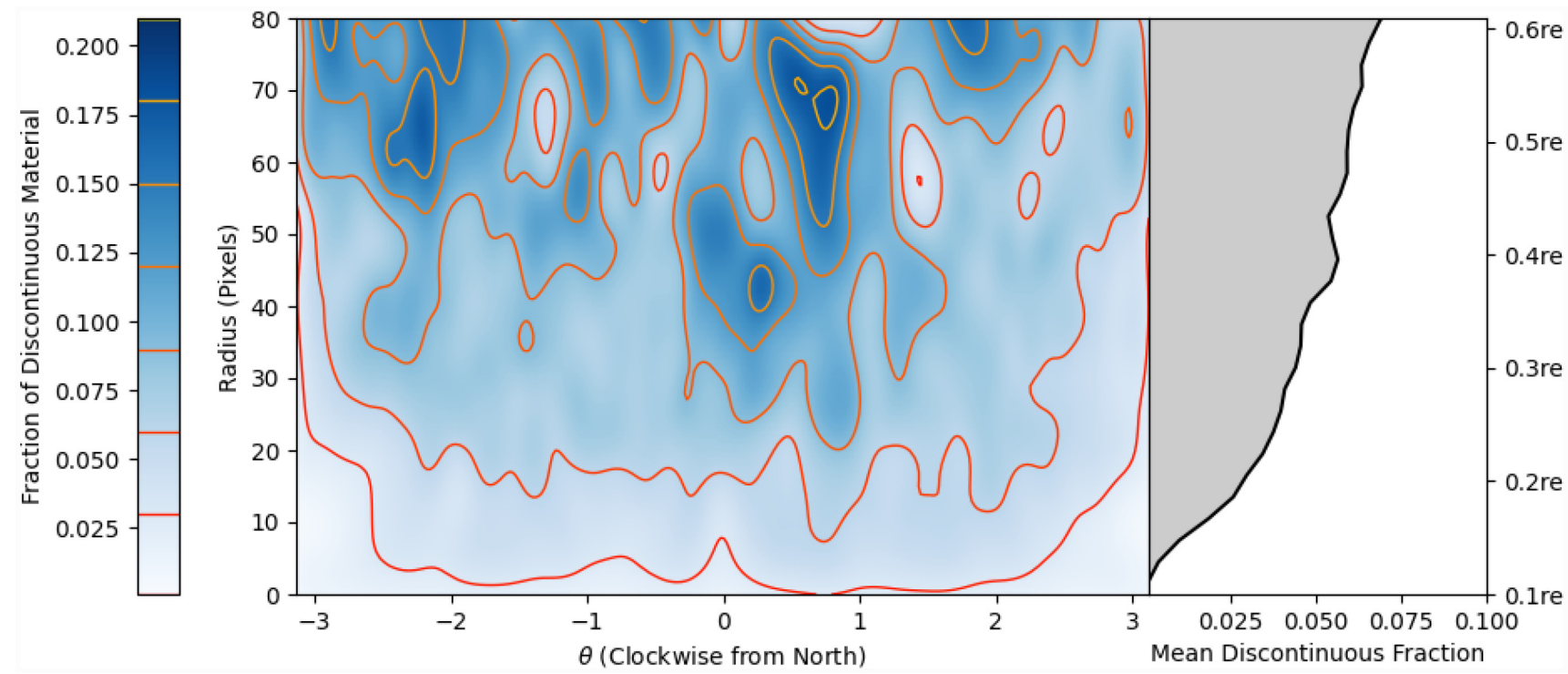

Figure 8. The left-hand panel shows a polar oriented map of NGC 7135. The blue colour shows the mass fraction of derived material not associated with the host galaxy population, with contouring shown in red-orange-yellow. The angle is shown with 0 radians as the North of the image and positive angle increase showing clockwise movement around the galaxy. Gaussian smoothing has been applied to show more clearly larger structures of ex-situ material. The radius from centre has been limited to include only radii in which a complete circle can be arranged within the image. The adjoining right-hand panel shows the same radial positions as the left-hand side, however it shows the mean discontinuous mass fraction for a complete circle for the radii. Mean fraction was calculated using circular annuli of radius 3 pixels with a moving average. The effective radius is taken from table 1 of Marino et al. (2011). The fraction of accreted material increases with radius, with a roughly 7 per cent increase within 0.6 effective radii. 

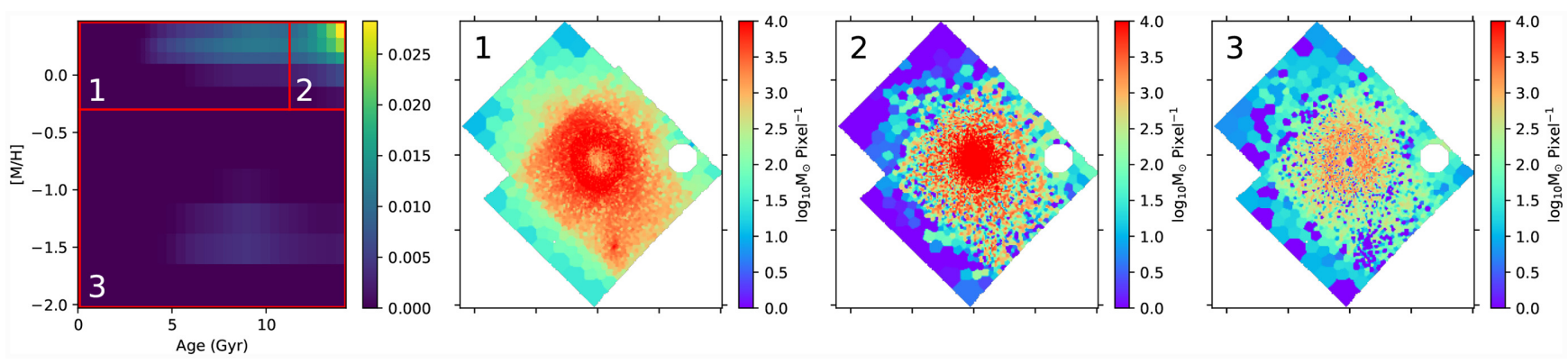

Figure 9. The first panel shows a general galaxy age-metallicity map. This is divided by the red boxes into three groups of populations to examine the mass associated with each area. Panel labels correspond to the numbers on the age-metallicity map. These show the divided nature of the populations, in which the intermediate age high metallicity population is more strongly associated with the infalling object and tidal features, whilst the older metal rich population is associated with the host galaxy.

figure (panel 2) in which there is a noticeably higher stellar mass associated with the infalling object for only this population. This panel also encompasses much of the stellar material of NGC 7135 near to the centre though at a slight distance, as is expected from standard galaxy age gradients. Though effects from the pointing overlaps are visible, it is notable that we see a small amount of material tracing the tidal tail and other tidally derived features. This suggests that the intermediate-age material and tidal tail is associated with the infalling galaxy exclusively, though further data analysis from a higher resolution stellar model grid would be required for verification of this.

In the second labelled map (panel 3) we see that the most metal rich and oldest material is associated heavily with the host galaxy, with a strong gradient from the galaxy centre. This in-situ population is generally undisturbed and centrally concentrated, in comparison to the largely ex-situ population represented in the first map. Finally in the third labelled map (panel 4), we see again a gradient of stellar mass associated with the host galaxy. This third map shows only stars at far lower metallicities than the majority of the stellar material. This material is assumed to be low mass objects which have historically accreted to NGC 7135, and are now well mixed into the galaxy. It should be noted that these are rigid divisions, and that the true population distributions from each object undoubtedly bleed over into the other divided regions (especially in regions ' 1 ' and ' 2 ').

\section{DISCUSSION}

Analysis of the galaxy kinematics and gas of NGC 7135 yielded evidence for both historical galaxy mergers, as well as an ongoing disruptive major merger. Despite the kinematics of past mergers being hidden (to the available resolution of data) due to mixing over time, ex-situ populations were extracted from the galaxy using full spectral fitting. This allowed for the identification of a well-mixed low-metallicity stellar population relative to the larger fraction of higher metallicity stellar population. Considering expected enrichment patterns, this can only have occurred if either gas or stars (or both) originating in an ex-situ galaxy rapidly accreted or fully merged on to NGC 7135. The lower metal content of this population made it distinct from the original population.

Potentially, all the stellar material in this population could have been created in-situ using gas that was accreted from another galaxy. This is highly unlikely however considering the specificity of the age and metallicity of the two distinct populations. Were these stars to be the product of new infalling gas, we would expect to see a mixing of the gas, and for the metallicity of new stars born after the merger event to be at a more intermediate metallicity. Instead, we see the two populations continuing to form stars without a sharp change in metallicity, thus the lower metallicity population stars are considered to be born ex-situ.

The bi-modality of these stars allowed for clean separation of the ex-situ and in-situ populations. Thus the relative fraction of ex-situ material could be ascertained. This allowed for the exploration of ex-situ fraction with galactocentric radius, as shown in Fig. 8. The figure shows a clear preference for ex-situ material to be located at the outer edges of the galaxy, with no detectable ex-situ material in the centre of the galaxy. This is akin to simulated results showing the same preference for ex-situ fraction increase with galactocentric radius (Schaye et al. 2014; Crain et al. 2015; Rodriguez-Gomez et al. 2016; Davison et al. 2020), as well as observational studies showing the same principles (Forbes et al. 2011; Pillepich, Madau \& Mayer 2015; Oyarzún et al. 2019). The mean ex-situ fraction measured for NGC 7135 at approximately 0.6 effective radii (the greatest extent captured by the MUSE image) is 7 per cent. This is only representative of the low metallicity populations from low-mass systems. Higher metallicity populations from mergers of smaller mass ratio mergers would be disguised amongst in-situ populations.

Limitations of this technique largely arise from the ability to separate populations. At current resolutions of full spectral fitting techniques, populations must be wholly distinct in metallicity to be noticeably separable from the host population. Accreted material with age and metallicity similar to that of the host galaxy would be largely indistinguishable from the main population. Further limitations are the inability to directly distinguish between stars that are born ex-situ, and those born in-situ but of ex-situ material. As discussed above, these limitations are unlikely to be dominant in this scenario.

One interesting area to consider is the eventual fate of NGC 7135. Will it retain some semblance of a spiral structure, or evolve into an S0 or elliptical galaxy? Conversion into an S0 galaxy seems to be a distinct possibility as S0 galaxies with coherent disc kinematics form through merger mechanisms, though the exact merger specifics continue to be debated within the community. Some evidence suggests that S0 galaxies are rarely expected to be formed through major mergers ( $<4: 1$ merger ratio) (Bournaud, Jog \& Combes 2005; Lofthouse et al. 2016), with the conclusion given that major mergers are a plausible but non-dominant mechanism for early-type formation. Conversely, other arguments suggest that S0 galaxies can indeed be formed from major mergers (Querejeta et al. 2015). Furthermore, major mergers can be shown to give rise to much of the inner structure often found in early types (Eliche-Moral 
et al. 2018). Perhaps the most consistent agreement for the formation requirements of an $\mathrm{S} 0$ via mergers is the necessity for a misalignment of angular momentum between the in-situ and ex-situ accreted baryonic components (see e.g. Sales et al. 2012). Considering the existing baryonic misalignment present in NGC 7135 in the form of a counter-rotating disc, and considering the seemingly misaligned orbit of the ongoing merger, it is perhaps likely that the ongoing disruption will lead to NGC 7135 tending towards S0 morphology. Plausibly the kinematics would increasingly reflect those of general spheroid galaxies as newly formed stars with an opposing angular momentum to the mean, and those recently accreted, would begin to reduce kinematic coherence. Though this is a distinct possibility, the true future of NGC7135 will remain unknown until more decisive techniques and modelling are developed. Due to the complex nature of the recent history of NGC 7135, any predictions on future evolution are speculation.

\section{CONCLUSIONS}

We have used a Voronoi binned map of NGC7135 to explore kinematic stellar features such as velocity and velocity dispersion, as well as the distributions of stellar properties such as age and metallicity. Gas properties were also explored in regular bins, with both kinematic gas properties and gas distribution investigated. Gas was shown to be counter-rotating compared to stellar material, with significant evidence of disturbance in the galaxy core. This along with population evidence shows a galaxy currently merging on to NGC 7135. Despite gas being present, little to no current star formation was identified. ALMA data of the galaxy core points to a star formation rate of only $0.025 \mathrm{M}_{\odot} \mathrm{yr}^{-1}$ assuming normal depletion times. Strong LINER emission likely obscures emission associated with star formation and as such a higher SFR cannot be ruled out.

During population analysis of NGC 7135 from data provided by the SOSIMPLE project, we have identified both historic and ongoing merger activity. This was achieved using a 'full spectral fitting' method to disentangle strong bi-modalities in stellar populations. We show that in a snapshot of a 'single' galaxy, we are in reality witnessing the product of three distinct galaxy populations.

An ongoing merger or large accretion event is clear from the stellar kinematic maps, showing a distinct area of stellar material not associated with the host galaxy, but interacting with the galaxy structure. Likewise in gas maps we see large velocity dispersion in areas where ex-situ infalling gas interacts with in-situ gas.

At least one historical large merger event took place at 6-10 Gyr ago according to star-formation history derived by full spectral fitting. This potentially provided gas with lower enrichment with which NGC 7135 birthed stars of lower metallicity; however the timeline of stellar ages, matched with the likely merger date makes it highly likely that most, if not all of the stars belonging to this population are ex-situ stars originating in another galaxy. Considering there is no discernible change in the host population metallicity of new stars born after the merger, we assume that all lower metallicity population stars are ex-situ in origin. The timeline of star formation history suggests that this merger caused a general shut-down of star formation in NGC 7135, not long after the merger event.

We calculate the fraction of the ex-situ material as a function of galactocentric radius, finding a steep increase in ex-situ material as we probe further to the outskirts of the galaxy. The centre of the galaxy exhibits no signs of ex-situ material, whilst by 0.6 effective radii, this fraction is at 7 per cent. This is in common with literature expectations of 'two phase' galaxy assembly seen both observationally and in simulation, where ex-situ material is preferentially deposited on the outskirts of a galaxy.

Many more SOSIMPLE galaxies are available from the survey, with much left to explore.

\section{ACKNOWLEDGEMENTS}

Many thanks to an anonymous referee for useful comments. This work was completed with the support of the ESO studentship program and the Moses Holden Scholarship. BH acknowledges support by the DFG (Deutsche Forschungsgemeinschaft) grant GE625/17-1 and DLR (Deutsches Zentrum für Luft- und Raumfahrt) grant 50OR1911. Based on observations collected at the European Southern Observatory under ESO programme 0103.A-0637(A).

\section{DATA AVAILABILITY}

The data described in this article are accessible via the ESO archive of MUSE data.

\section{REFERENCES}

Annibali F., Bressan A., Rampazzo R., Zeilinger W., Vega O., Panuzzo P., 2010, A\&A, 519, A40

Bacon R. et al., 2010, in Ground-based and Airborne Instrumentation for Astronomy III, SPIE, p. 773508

Bacon R. et al., 2014, Messenger, 157, 13

Baldwin J. A., Phillips M. M., Terlevich R., 1981, PASP, 93, 5

Barr J., Bedregal A., Aragón-Salamanca A., Merrifield M., Bamford S., 2007, A\&A, 470, 173

Barrera-Ballesteros J. et al., 2015, A\&A, 582, A21

Bassett R., Bekki K., Cortese L., Couch W., 2017, MNRAS, 471, 1892

Bekki K., 2001, Astrophys. Space Sci., 276, 847

Bertola F., Buson L., Zeilinger W. W., 1988, Nature, 335, 705

Bigiel F. et al., 2011, ApJ, 730, L13

Boecker A., Alfaro-Cuello M., Neumayer N., Martín-Navarro I., Leaman R., 2020, ApJ, 896, 13

Bournaud F., Jog C., Combes F., 2005, A\&A, 437, 69

Bryant J. et al., 2019, MNRAS, 483, 458

Bundy K., Fukugita M., Ellis R. S., Targett T. A., Belli S., Kodama T., 2009, ApJ, 697, 1369

Cales S. L., Brotherton M. S., 2015, MNRAS, 449, 2374

Capelo P. R., Dotti M., 2017, MNRAS, 465, 2643

Capelo P. R., Volonteri M., Dotti M., Bellovary J. M., Mayer L., Governato F., 2015, MNRAS, 447, 2123

Cappellari M., 2017, MNRAS, 466, 798

Cappellari M., Emsellem E., 2004, PASP, 116, 138

Carraro G., Chiosi C., 1994, A\&A, 287, 761

Cassisi S., Pietrinferni A., Salaris M., Castelli F., Cordier D., Castellani M., 2006, Mem. Soc. Astron. Ital., 77, 71

Choi E., Ostriker J. P., Naab T., Oser L., Moster B. P., 2015, MNRAS, 449, 4105

Coccato L. et al., 2015, A\&A, 581, A65

Comerón S., Salo H., Janz J., Laurikainen E., Yoachim P., 2015, A\&A, 584, A34

Cortesi A. et al., 2013, MNRAS, 432, 1010

Crain R. A. et al., 2015, MNRAS, 450, 1937

Daddi E. et al., 2005, ApJ, 626, 680

Davis T. A. et al., 2011, MNRAS, 417, 882

Davison T. A., Norris M. A., Pfeffer J. L., Davies J. J., Crain R. A., 2020 MNRAS, 497, 81

Di Matteo P., Bournaud F., Martig M., Combes F., Melchior A.-L., Semelin B., 2008, A\&A, 492, 31

Ebeling H., Stephenson L. N., Edge A. C., 2014, ApJ, 781, L40

Eliche-Moral M. d. C., Rodríguez-Pérez C., Borlaff A., Querejeta M., Tapia T., 2018, A\&A, 617, A113 
Ellison S. L., Mendel J. T., Scudder J. M., Patton D. R., Palmer M. J., 2013, MNRAS, 430, 3128

Faifer F. R., Escudero C. G., Scalia M. C., Smith Castelli A. V., Norris M., De Rossi M. E., Forte J. C., Cellone S. A., 2017, A\&A, 599, L8

Fakhouri O., Ma C.-P., Boylan-Kolchin M., 2010, MNRAS, 406, 2267

Falcón-Barroso J., Sánchez-Blázquez P., Vazdekis A., Ricciardelli E., Cardiel N., Cenarro A., Gorgas J., Peletier R., 2011, A\&A, 532, A95

Forbes D. A., Spitler L. R., Strader J., Romanowsky A. J., Brodie J. P., Foster C., 2011, MNRAS, 413, 2943

Ge J., Mao S., Lu Y., Cappellari M., Yan R., 2019, MNRAS, 485, 1675

Guérou A., Emsellem E., Krajnović D., McDermid R. M., Contini T., Weilbacher P. M., 2016, A\&A, 591, A143

Ho L. C., Li Z.-Y., Barth A. J., Seigar M. S., Peng C. Y., 2011, ApJS, 197, 21

Husser T.-O. et al., 2016, A\&A, 588, A148

Keel W. C., 1985, AJ, 90, 2207

Kennicutt R. C., Jr, 1998, ApJ, 498, 541

Kroupa P., 2001, MNRAS, 322, 231

L'Huillier B., Combes F., Semelin B., 2012, A\&A, 544, A68

La Barbera F., Ferreras I., de Carvalho R. R., Bruzual G., Charlot S., Pasquali A., Merlin E., 2012, MNRAS, 426, 2300

Layden A. C., Sarajedini A., 2000, AJ, 119, 1760

Leaman R., VandenBerg D. A., Mendel J. T., 2013, MNRAS, 436, 122

Leroy A. K. et al., 2013, AJ, 146, 19

Lofthouse E., Kaviraj S., Conselice C. J., Mortlock A., Hartley W., 2016, MNRAS, 465, 2895

Lotz J. M., Jonsson P., Cox T., Croton D., Primack J. R., Somerville R. S., Stewart K., 2011, ApJ, 742, 103

Malin D., Carter D., 1983, ApJ, 274, 534

Maller A. H., Katz N., Kereš D., Davé R., Weinberg D. H., 2006, ApJ, 647, 763

Marino A. et al., 2011, MNRAS, 411, 311

Martin G., Kaviraj S., Devriendt J. E. G., Dubois Y., Pichon C., 2018, MNRAS, 480, 2266

Mihos C., Hernquist L., 1995, preprint (astro-ph/9512099)

Monreal-Ibero A., Arribas S., Colina L., Rodríguez-Zaurín J., Alonso-Herrero A., García-Marín M., 2010, A\&A, 517, A28

Moreno J., Torrey P., Ellison S. L., Patton D. R., Bluck A. F., Bansal G., Hernquist L., 2015, MNRAS, 448, 1107

Norris M. A., Escudero C. G., Faifer F. R., Kannappan S. J., Forte J. C., van Den Bosch R. C., 2015, MNRAS, 451, 3615

Oyarzún G. A. et al., 2019, ApJ, 880, 111

Park S., Sohn B. W., Sukyoung K. Y., 2013, A\&A, 560, A80

Pietrinferni A., Cassisi S., Salaris M., Castelli F., 2006, ApJ, 642, 797
Pillepich A., Madau P., Mayer L., 2015, ApJ, 799, 184

Pillepich A. et al., 2018, MNRAS, 475, 648

Pont F., Eyer L., 2004, MNRAS, 351, 487

Puglisi A. et al., 2021, Nat. Astron.

Qu Y. et al., 2017, MNRAS, 464, 1659

Querejeta M. et al., 2015, A\&A, 579, L2

Quilis V., Trujillo I., 2013, ApJ, 773, L8

Rampazzo R., Plana H., Longhetti M., Amram P., Boulesteix J., Gach J. L., Hernandez O., 2003, MNRAS, 343, 819

Rampazzo R. et al., 2007, MNRAS, 381, 245

Rich J. A., Kewley L. J., Dopita M. A., 2011, ApJ, 734, 87

Rodriguez-Gomez V. et al., 2016, MNRAS, 458, 2371

Rubin V. C., 1994, AJ, 108, 456

Sales L. V., Navarro J. F., Theuns T., Schaye J., White S. D., Frenk C. S., Crain R. A., Dalla Vecchia C., 2012, MNRAS, 423, 1544

Samir R., Reda F., Shaker A., Osman A., Amin M., 2016, NRIAG J. Astron. Geophys., 5, 277

Saracco P., Longhetti M., Andreon S., 2009, MNRAS, 392, 718

Schawinski K., Dowlin N., Thomas D., Urry C. M., Edmondson E., 2010, ApJ, 714, L108

Schaye J. et al., 2014, MNRAS, 446, 521

Shanks T. et al., 2015, MNRAS, 451, 4238

Sinha M., Holley-Bockelmann K., 2012, ApJ, 751, 17

Solomon P., Barrett J., 1991, in Symposium-International Astronomical Union. p. 235

Spolaor M., Proctor R. N., Forbes D. A., Couch W. J., 2009, ApJ, 691, L138

Thomas D., Maraston C., Bender R., 2003, MNRAS, 339, 897

Trujillo I., Ferré-Mateu A., Balcells M., Vazdekis A., Sánchez-Blázquez P., 2013, ApJ, 780, L20

Tully R. B., Fisher J. R., 1988, Nearby galaxies catalog. Cambridge Univ. Press, Cambridge

Ueda J. et al., 2014, ApJS, 214, 1

Van Dokkum P. G. et al., 2008, ApJ, 677, L5

Vazdekis A., Sánchez-Blázquez P., Falcón-Barroso J., Cenarro A., Beasley M., Cardiel N., Gorgas J., Peletier R., 2010, MNRAS, 404, 1639

Vazdekis A., Ricciardelli E., Cenarro A., Rivero-González J., Díaz-García L., Falcón-Barroso J., 2012, MNRAS, 424, 157

Ventou E. et al., 2017, A\&A, 608, A9

Weilbacher P. M. et al., 2020, A\&A, 641, A28

Zaw I., Farrar G. R., Greene J. E., 2009, ApJ, 696, 1218

This paper has been typeset from a $\mathrm{T}_{\mathrm{E}} \mathrm{X} / \mathrm{L} \mathrm{T} \mathrm{E} \mathrm{X}$ file prepared by the author. 